\title{
Research Paper: The Relationship of Spiritual Intelligence and Rehabilitation Staff's Perceived Stress in Welfare Organizations Centers in Tehran and Shemiranat: 2017
}

\section{Banafsheh Ebrahimi Barmi ${ }^{1}{ }^{\oplus},{ }^{*}$ Mohammadali Hosseini ${ }^{1} \oplus$, Kianoush Abdi $^{1}$, Enayatollah Bakhshi²}

1. Department of Rehabilitation Management, University of Social Welfare and Rehabilitation Sciences, Tehran, Iran.

2. Department of Biostatistics, University of Social Welfare and Rehabilitation Sciences, Tehran, Iran.

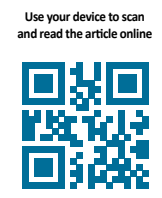

ditat on Ebrahimi Barmi B, Hosseini M, Abdi K, Bakhshi E. [The Relationship of Spiritual Intelligence and Rehabilitation Staff's Perceived Stress in Welfare Organizations Centers in Tehran and Shemiranat: 2017 (Persian)]. Archives of Rehabilitation. 2018; 19(3):228-237. http://dx.doi.org/10.32598/rj.19.3.228

dol: : http://dx.doi.org/10.32598/rj.19.3.228

Keywords:

Spiritual intelligence, Perceived stress, Rehabilitation staff

\section{ABSTRACT}

Objective Today, one of the factors that threatens the mental health of the employees is the stress of their organizations. The nature and essence of the medical organizations are such that these environments inevitably impose stress on employees. Perceived stress at work affects the quality and performance of an employee. Knowing how to cope with perceived stress can be effective in preventing and reducing complications. The rehabilitation work environments are in high stress and this stress can exacerbate the quality of the work of experts and ultimately affect the health of staff. This study aimed to determine the relation of spiritual intelligence with rehabilitation staff's perceived stress in welfare organization centers in Tehran City and Shemiran County, Iran in 2017.

Materials \& Methods This research is a correlational and cross-sectional study. A total of 163 rehabilitation experts (in different fields of physiotherapy, occupational therapy, speech therapy, audiology, optometry, technical orthopedics, and psychology) worked in welfare organization centers of Tehran City and Shemiran County were selected by random cluster sampling method in 2017 . In order to collect data, a demographic questionnaire, Spiritual Intelligence Questionnaire (2008) (Cronbach $\alpha=0.88$ ) and perceived stress Cohen (1983) (Cronbach $\alpha=0.84$ ) were used. The validity and reliability of the tools used in this research have been approved by other researchers in similar studies. To analyze the data, SPSS version 21 was used. Descriptive statistics such as mean and standard deviation were used to describe the data. Regarding the normal distribution of data, Pearson correlation coefficient was used to analyze the data. The Independent $t$ test was used to compare the two groups data.

Results The average age of the study participants was 40 years, ranged from 23 to 54 years. About $56.5 \%$ of the participants in this study were female and $22.9 \%$ had a work experience of 10-15 years. The results showed no relationship between age, sex, work experience, marital status, education and type of employment with spiritual intelligence and perceived stress. However, there was a significant correlation between spiritual intelligence and perceived stress $(P=0.042)$.

Conclusion According to the findings, people with high perceived stress, have low spiritual intelligence. In order to boost spiritual intelligence and reduce the perceived stress of individuals, it is recommended that spiritual intelligence be taught to employees at regular intervals. Since the researcher has used the questionnaire as the only measurement tool in collecting data, it is necessary to be cautious in interpreting the results. Therefore, for more comprehensive research, techniques such as viewing, interviewing, and analyzing content are recommended.

\section{* Corresponding Author:}

Mohammadali Hosseini, PhD

Address: Department of Rehabilitation Management, University of Social Welfare and Rehabilitation Sciences, Tehran, Iran.

Tel: +98 (21) 22180132

E-Mail: mahmaimy2020@gmail.com 


\title{
رابطه هوش معنوى با استرس دركشده كارشناسان توانبخشى مراكز توانبخشى وابسته به

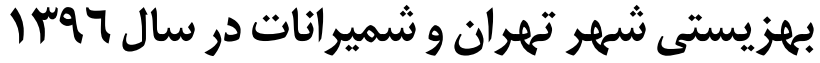

\author{
بنفشه ابراهيمى برمى' (1)، "محمدعلى حسينى' (1)، كيانوش عبدى'، عنايتالله بخشى'

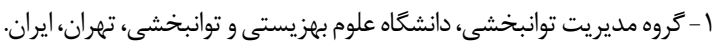

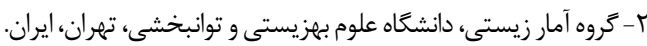

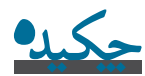

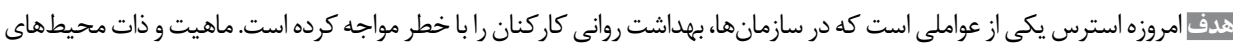

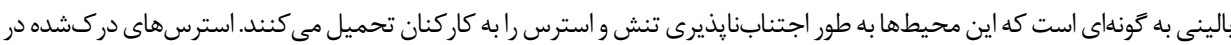

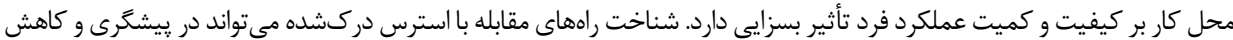

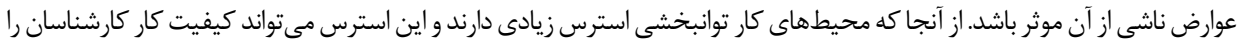

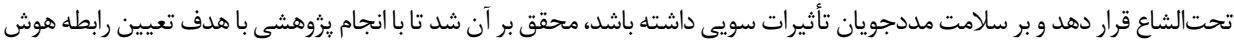

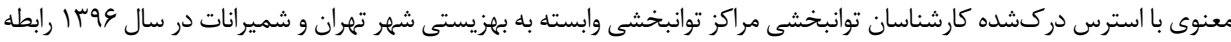

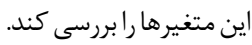

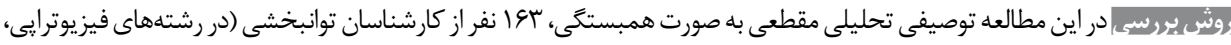

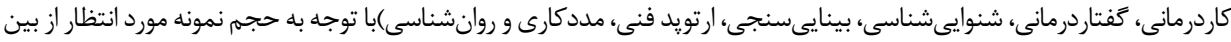

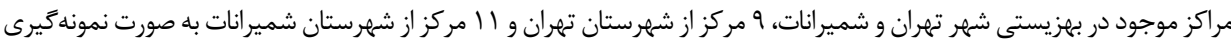

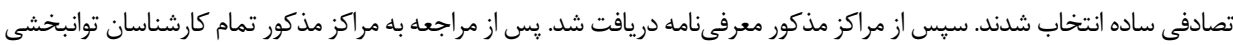

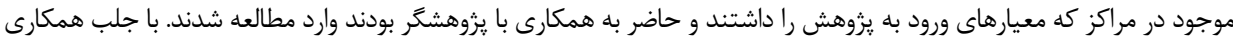

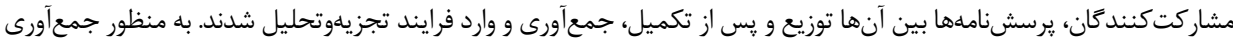

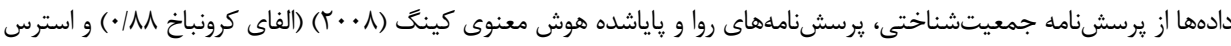

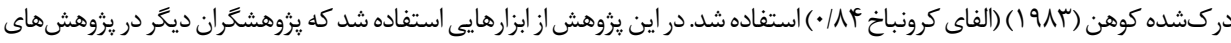

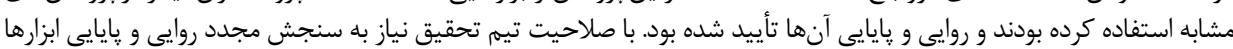

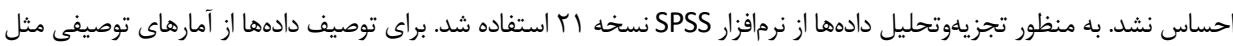

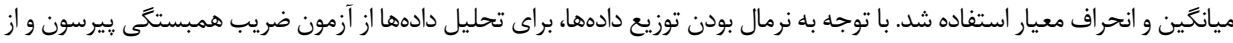

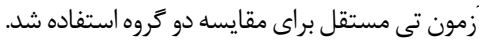

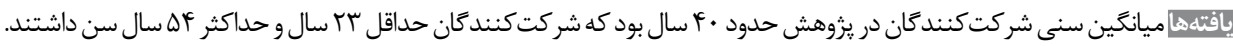

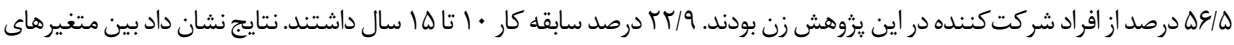

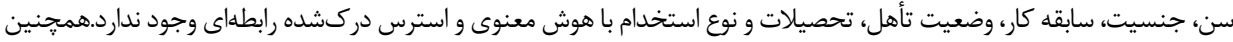

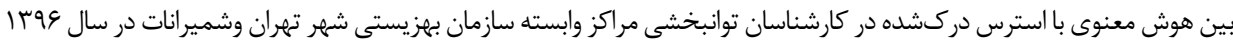

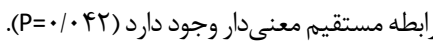

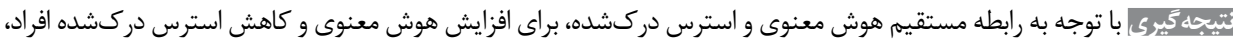

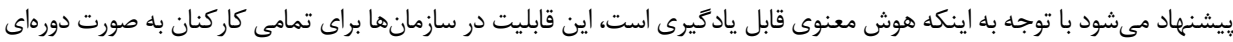

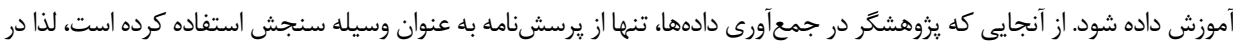

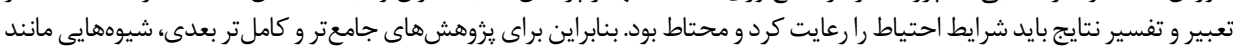

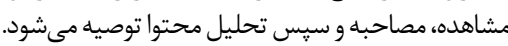

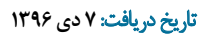

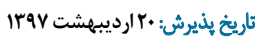

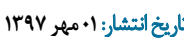

كليدوأرهها:

هوش معنوى، استرس

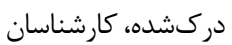
توانبخشى درك كثن 


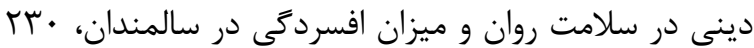

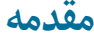

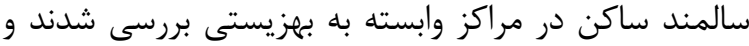

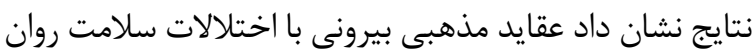
ارتباط دارد [• [1].

مذهب به دليل نقش مقابلهاى در برابر فشارهاى روانى

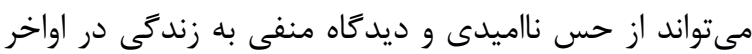

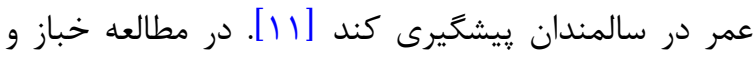

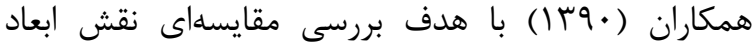

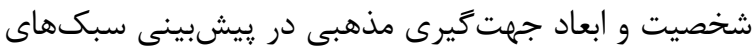

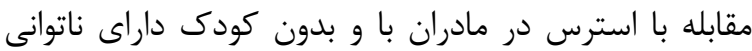

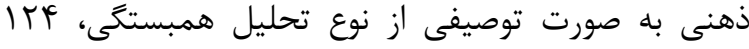

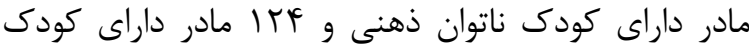

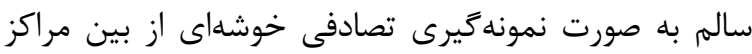

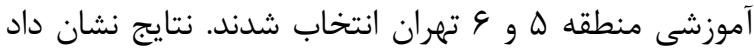

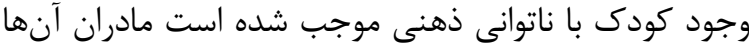

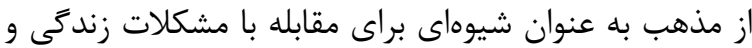

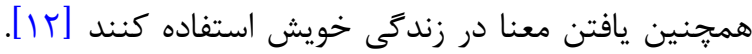

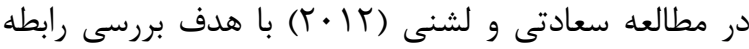

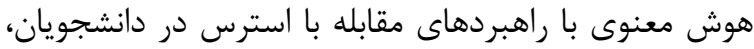

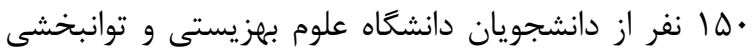

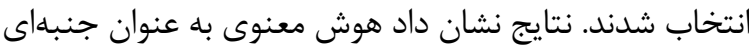

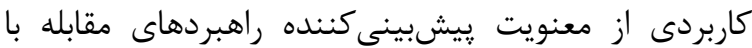

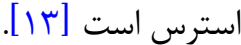

اهميت و ضرورت طرح موضوع معنويت و مذهب، بلويزه

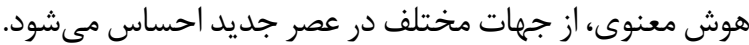

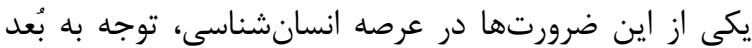

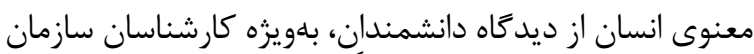

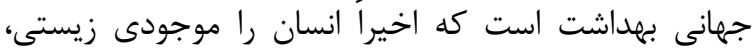

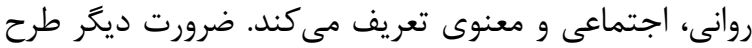

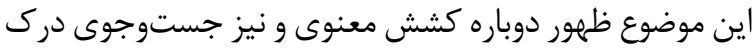

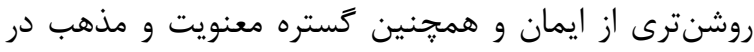

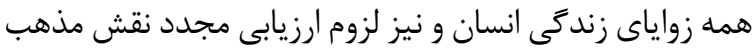

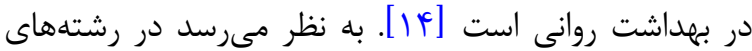

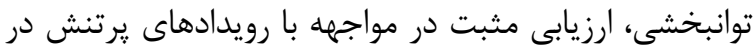

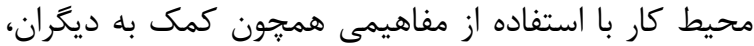

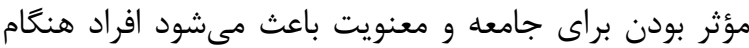

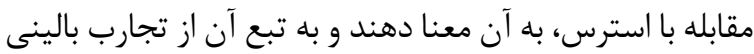

خود رضايت بيشترى داشته باشند [هاند إن]

از آنجا كه كارشناسان توانبخشى با سلامت افراد جامعهد

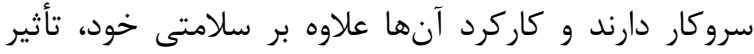

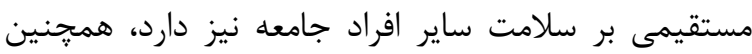

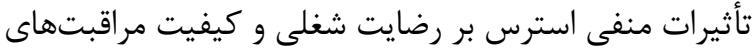

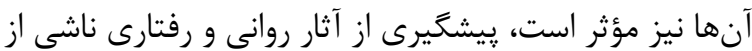

ارتقاى بهداشت روانى محيط كار از مهمترين ابعاد توسعه

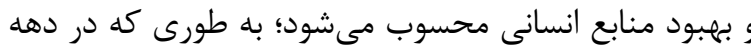

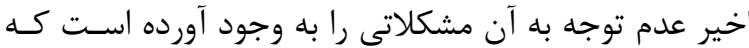

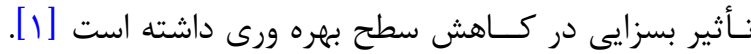

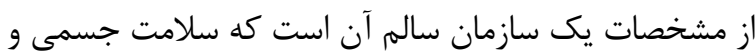

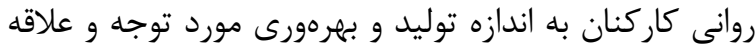

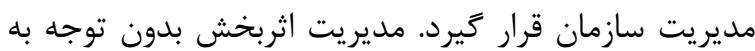

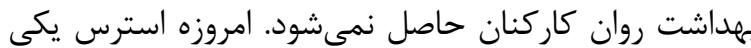

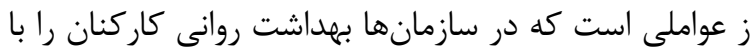

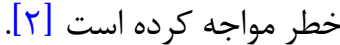
با توجه به اينكه اكثر كارشناسان منتخب در بخشهاى

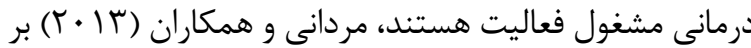

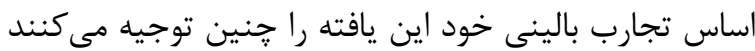

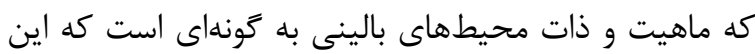

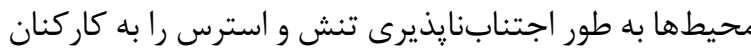

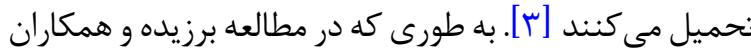

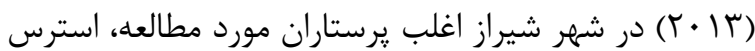

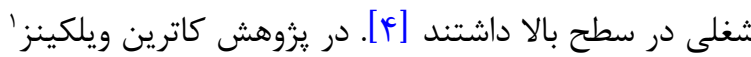

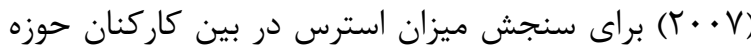

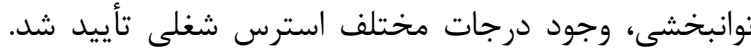

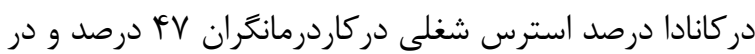

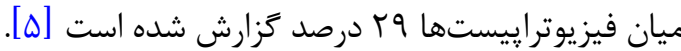
به نظر مىرسد مذهب براى افراد نقش سير دفاعى را ايفا و

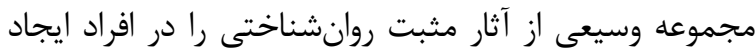

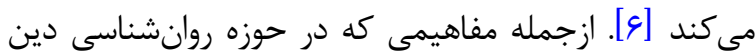

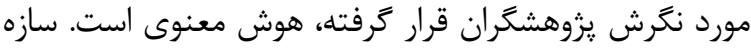

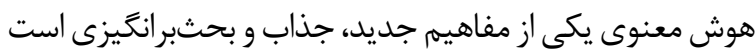

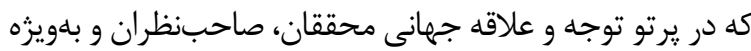

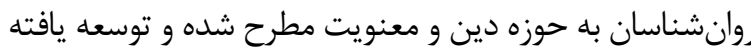

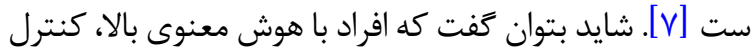

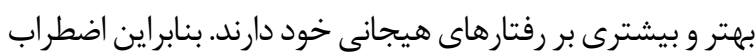

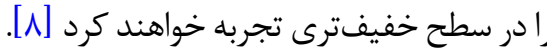

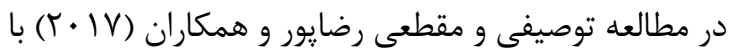

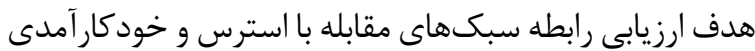

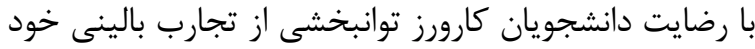

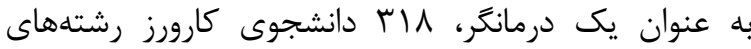

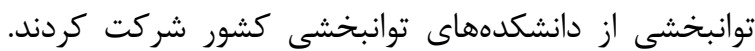

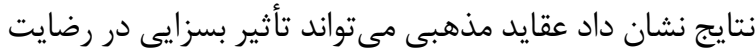

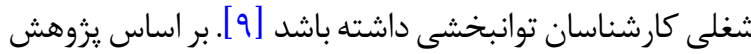

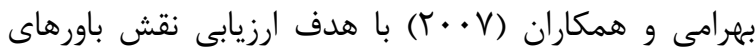

1. Wilkins 


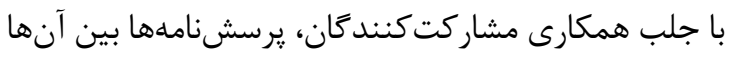

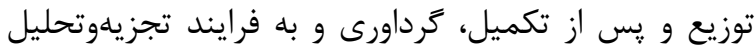

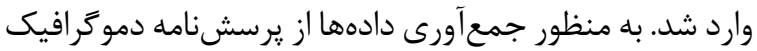

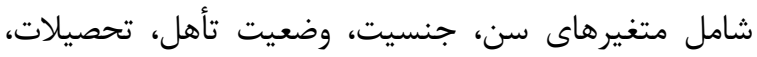

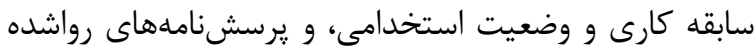

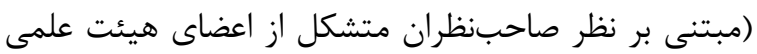

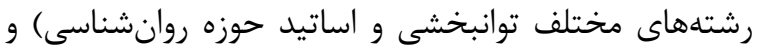

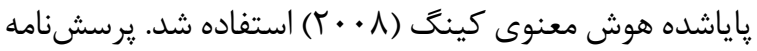

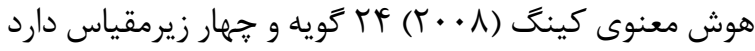

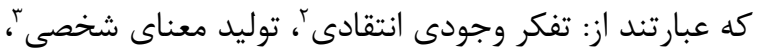

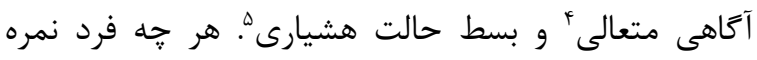
بيشترى در اين يرسشنامه بخيرد هوش معنوى بيشترى دارد.

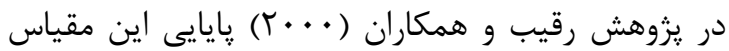

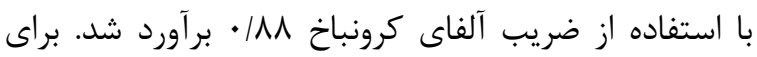

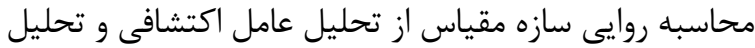

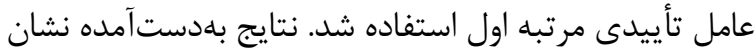

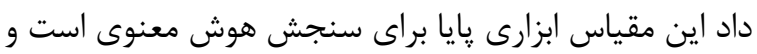

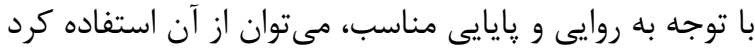

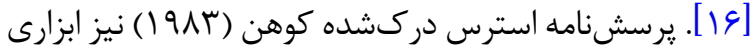

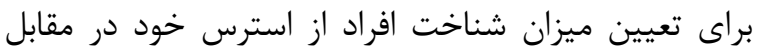

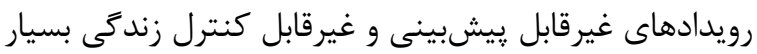

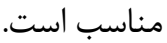

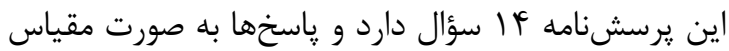

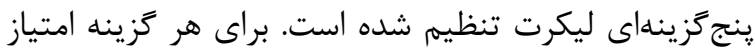

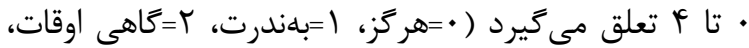

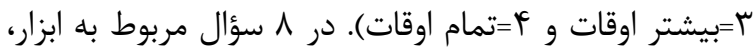

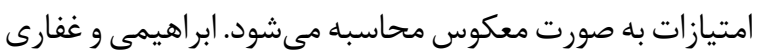

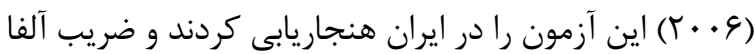

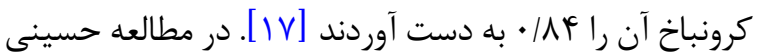

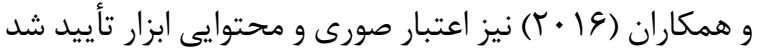

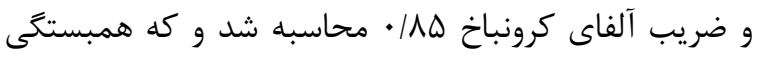

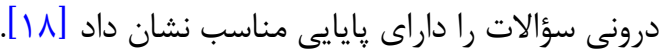

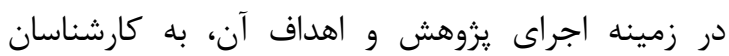

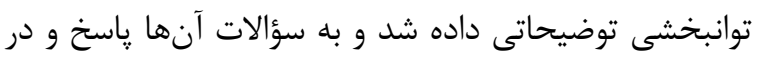

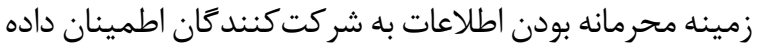

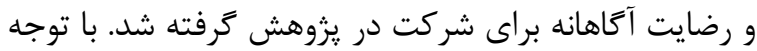

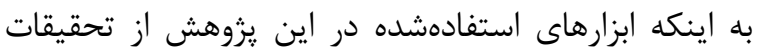

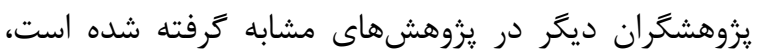
روايى و يايايى آنها تأييد شده است. بأ صلاحديد تيم تحقيق

2. Critical existential thinking

3. Personal meaning production

4. Transcendental awareness

5. Conscious state expansion

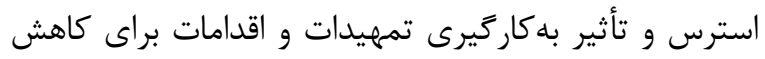

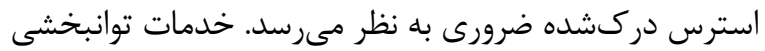

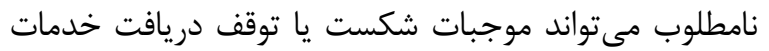

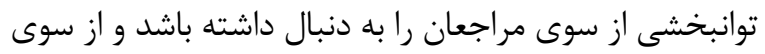

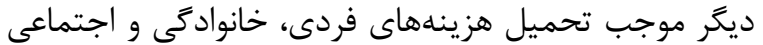
به نظام ارائه خدمات توانبخشي هزينه شود. با جستوجو در يايغاههاى اطلاعاتى (ايرانى و خارجى)

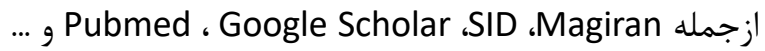
با كليدوازههاى مربوطه مانند هوش معنوى، استرس درك إنشده،

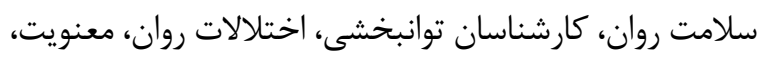

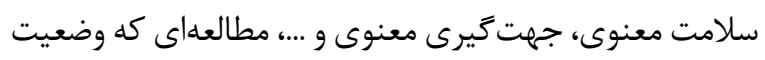

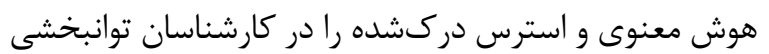

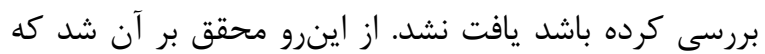

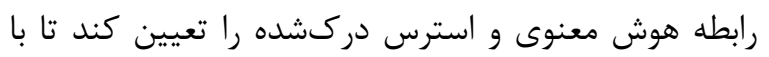

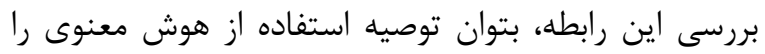
براى كاهش استرس دركشده اين افران توصيه استفاده ائه كرد.

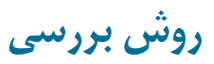

اين مطالعه توصيفى تحليلى مقطعى و به صورت همبستخى فرانى

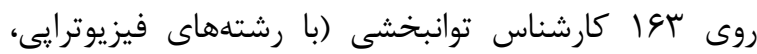

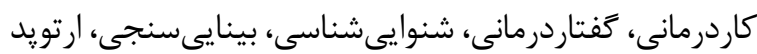

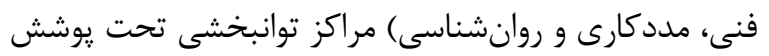

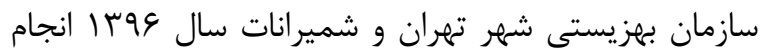

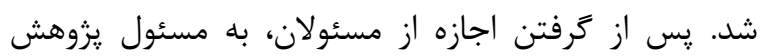

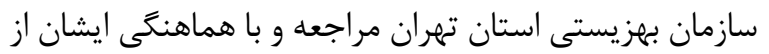

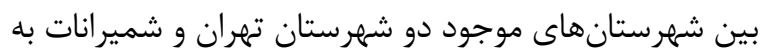

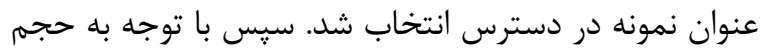

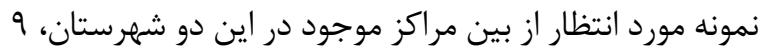

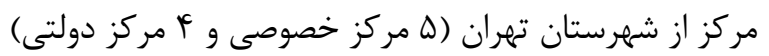

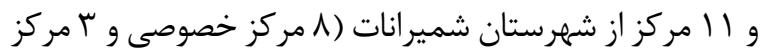

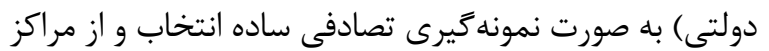
مذكور معرفىنامه دريافت شد.

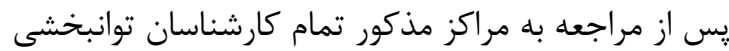

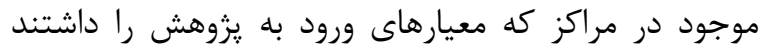

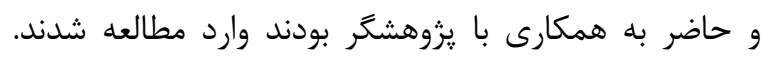

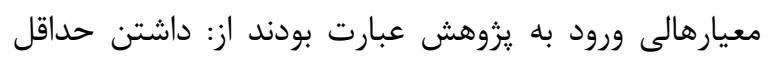

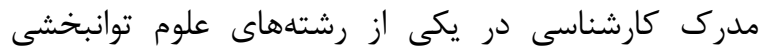

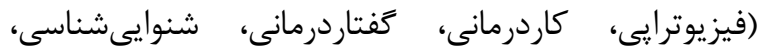

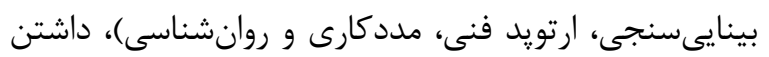

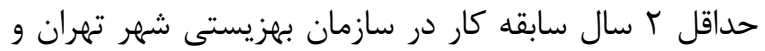

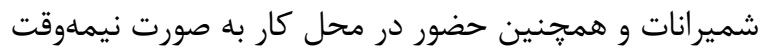

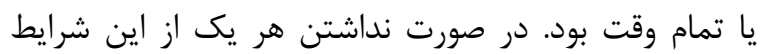
نمونهها از مطالعه حذف شدن مرند. 
جدول ا. توزيع خردهمقياسهاى هوش معنوى

\begin{tabular}{|c|c|c|c|c|c|}
\hline حداكثر نمره افراد & حداقل نمره افراد & انحراف معيار & ميانكين & تعداد & خرده مقياس \\
\hline rq & r & D/.r & $|9 / 9|$ & IST & تفكر وجودى انتقادى \\
\hline 19 & 9 & T/QF & $\mid r / \Delta T$ & I TI & توليد معناى شخصى \\
\hline rq & $\Delta$ & $F / 99$ & $|\varepsilon| \cdot r$ & سو & آَكاهى متعالى \\
\hline IV & 1 & r/q & $q / / r$ & I & بسط حالت هشيارى \\
\hline 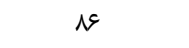 & r & $\mid F / \& V$ & $\Delta \psi / \varepsilon$. & I & نمره كلى \\
\hline
\end{tabular}

\begin{tabular}{|c|c|c|c|c|c|}
\hline حداكثر نمره افراد & حداقل نمره افراد & انحراف معيار & ميانكين & تعداد & متغير متير \\
\hline pr & ra & $r / \mu r$ & $r r / q$. & سצו & رس دركشده \\
\hline
\end{tabular}

توانبخننى

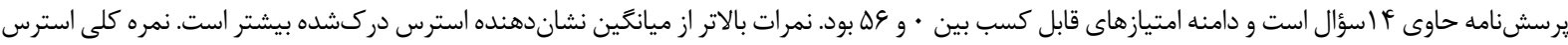

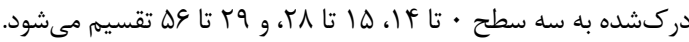

حالت هوشيارى بايينتر از ميانگَين است. نتايج نشان داد بين

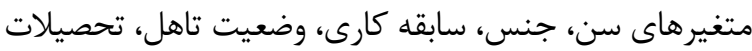
و نوع استخدام با هوش معنوى رابطه اى وجود ندائ ندارد. نتايج جدول شماره r نشان داد كارشناسان توانبخشى مراكز

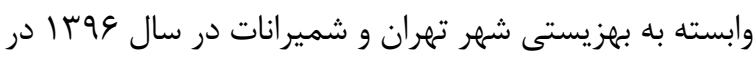

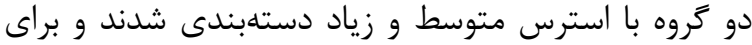

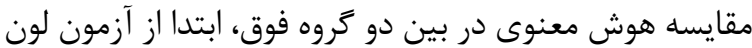

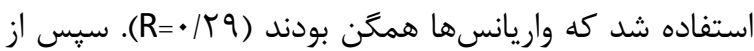

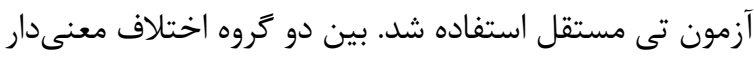

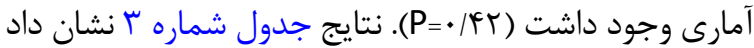

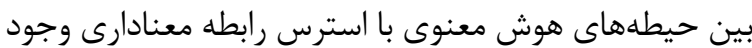

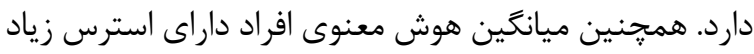
كمتر از افراد داراى استرس متوسط است.

بحث

يزوهش حاضر با هدف تعيين رابطه هوش معنوى با استرس تراس

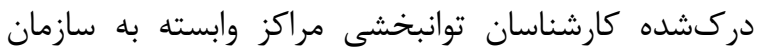

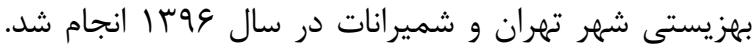

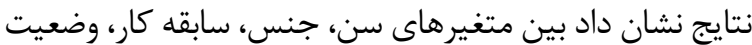

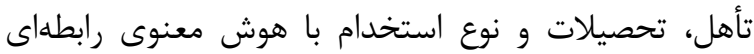

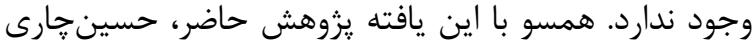

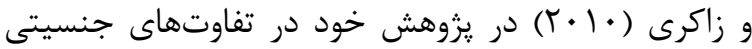

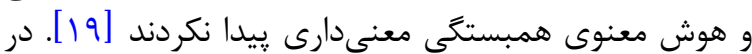

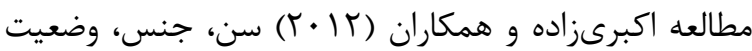

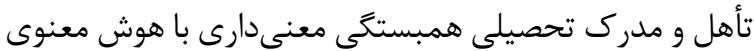

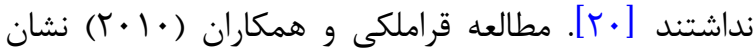

نياز به سنجش مجدد روايى و يايايى ابزارها احساس نشد.

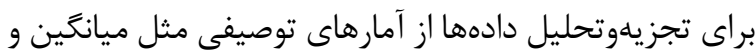

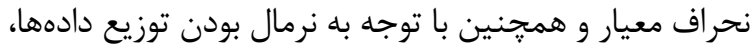

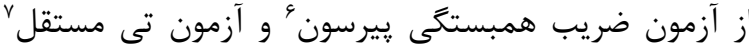
براى مقايسه دوخروه استفاده شد.

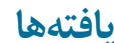

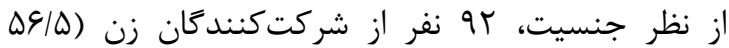

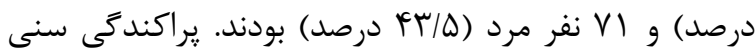
شركت كنند

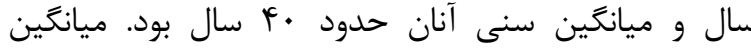
سابقه كار شركتكنيند

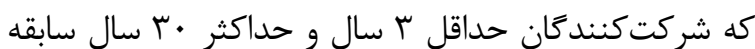

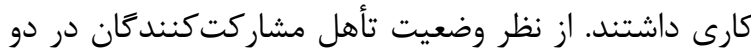

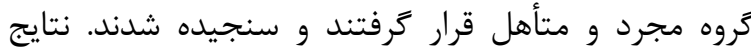

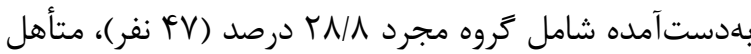

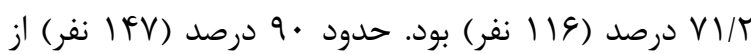

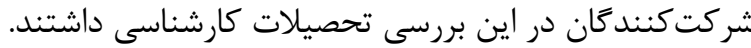

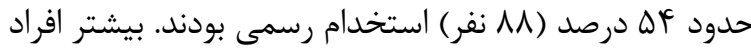

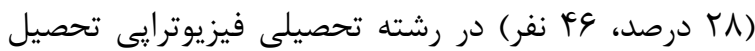

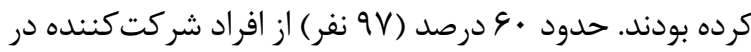
اين يزوهش در مراكز خصوصى مشغول به كار بودند. با توجه به جدول شماره ا ابعاد تفكر وجودى انتقادى، توليد

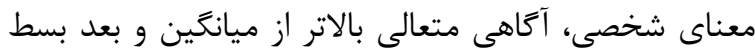


جدول س. مقايسه ميانگين نمرات هوش معنوى در سطوح مختلف استرس

\begin{tabular}{|c|c|c|c|c|}
\hline مقدار احتمال" & انحراف معيار & ميانكَين & تعداد & سطوح استرس \\
\hline & $\mid r / \pi \Delta$ & $g r / \Delta F$ & זו & متوسط \\
\hline \multicolumn{5}{|l|}{ H. } \\
\hline & $15 / 9 q$ & $\Delta r / 91$ & 10. & زياد \\
\hline
\end{tabular}

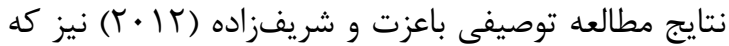

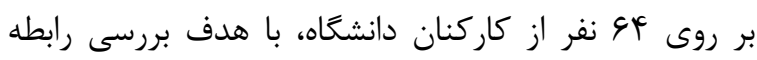
هوش معنوى و هوش هيجانى با استرس كاركنان دانشعاه

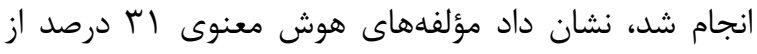

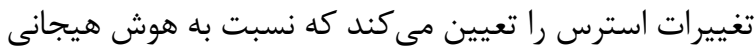

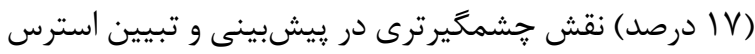

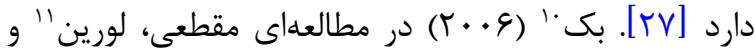

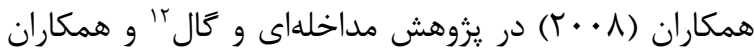

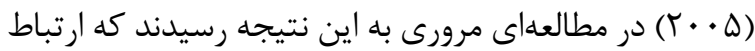
مؤثر با خدا و هوش معنوى باعث كاهش استرس، اضطراب و و

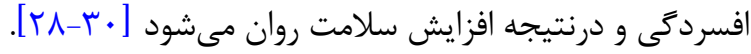

هنگامى كه فرد براى مقابله با فشارهاى زندگى به كمكى

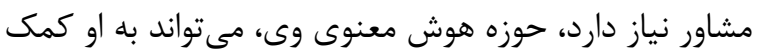

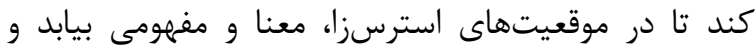

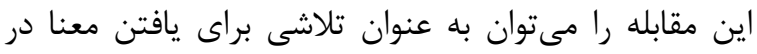

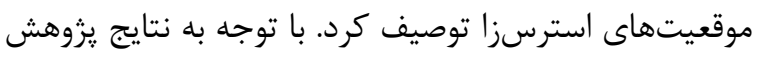

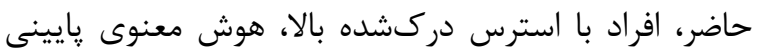

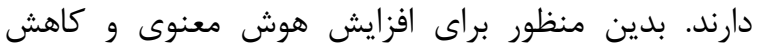

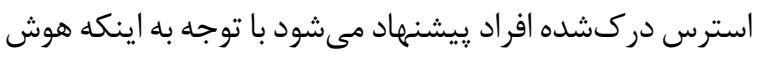

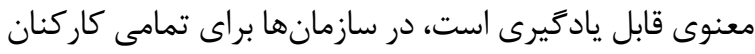

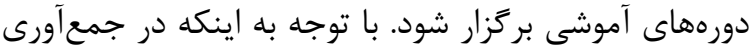

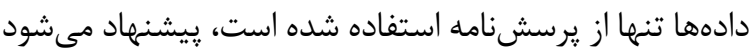
مطالعات كيفى انجام شود.

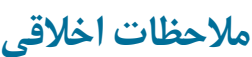

\section{يبروى از اصول اخالاق يُووهش}

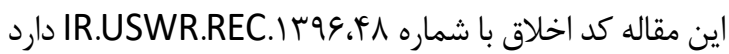

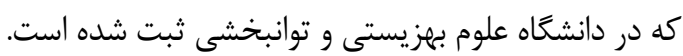

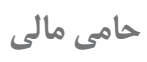

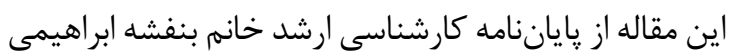

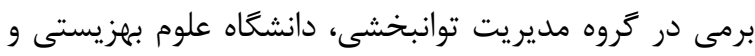
توانبخشى گرفته شده است.

10. Beck

11. Laurin

12. Gall
داد هوش معنوى دانشجويان متأهل بالاتر از مجردان و رابطه

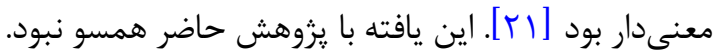
نتايج گروهبندى اعمالشده توسط تيم تحقيق نشان داد

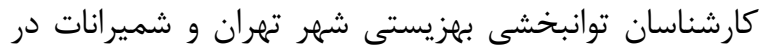

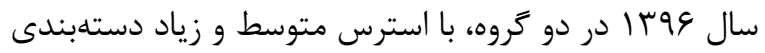
شدند و بين متغيرهاى سن، جنس، سابقه كارى، وضعيت

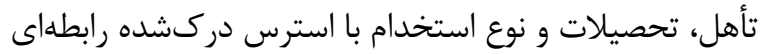

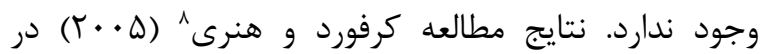

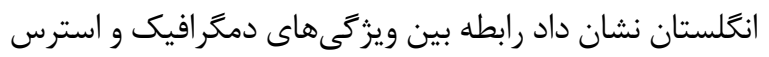

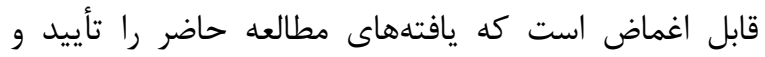

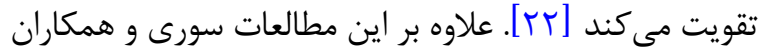

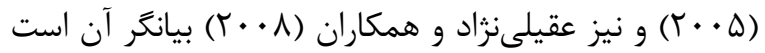

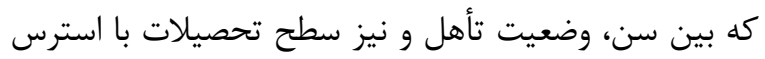

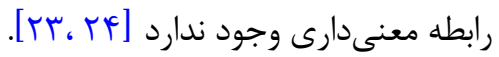

هاينز" (1994 (19) در مطالعه خود نشان داد بين سن و استرس،

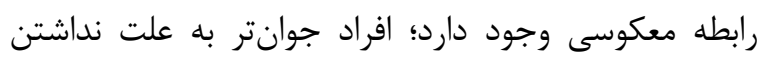

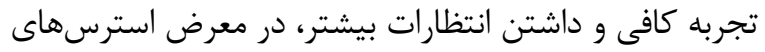

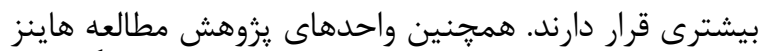

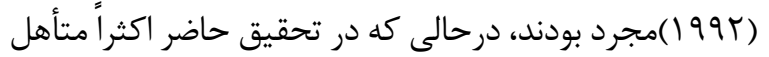

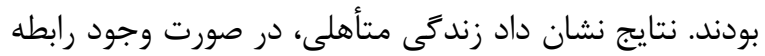

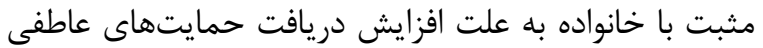

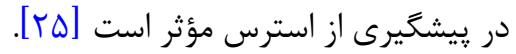
نتيجحه تيرى

نتايج نشان داد بين حيطههاى هوش معنوى با استرس معن مارى

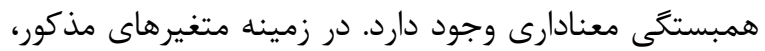
تحقيقات فراوانى صورت گرفته است. لواسانى و همكاران

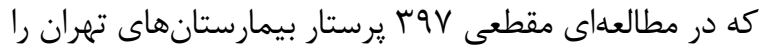

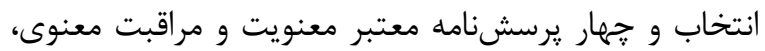

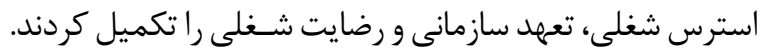

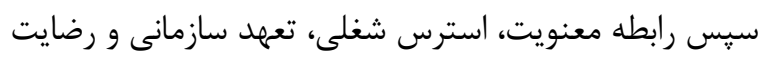

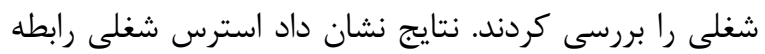

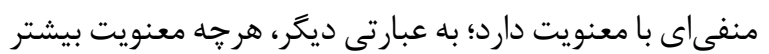

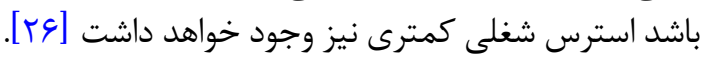

8. Crawford and Henry

9. Hains 


$$
\text { تعارض منافع }
$$

اين مقاله هيجَّونه تعارض منافع مالى مستقيم و غيرمستقيم و تعارض حرفهاى با كسى يا جايى ندارد.

$$
\text { تشكر و قدردانى }
$$

از معاونت توانبخشى بهزيستى استان تهران، شهر تهران و

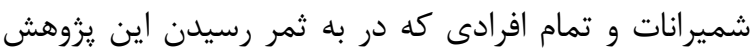

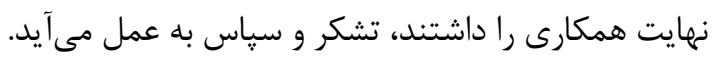




\section{References}

[1] Mahmoodi A, TabatabaeiNasab SE. [Investigation of the relationship between mental health and organizational employees' work fatigue and deputyships of Yasouj Medical Science University (Persian)]. Armaghan-e Danesh. 2015; 20(5):444-52.

[2] Faraji A, Valiee S, Mazidi G, Ramazanh A, Rezaee Farahani M. [Relationship between job characteristic and job stress in nurses of Kurdistan University of Medical Sciences educational hospitals (Persian)]. Iranian Journal of Nursing Research. 2012; 7(25):54-63.

[3] Mardani Hamooleh M, Ebrahimi E, Mostaghasi M, Taghavi Larijani T. [Relationship between organizational justice and job stress among hospital personnel (Persian)]. Iranian Journal of Medical Ethics and History of Medicine. 2013; 6(3):64-71.

[4] Barzideh M, Choobineh A, Tabatabaee SH. Job stress dimensions and their relationship to general health status in nurses. Work. 2014; 47(4):423-9. [DOI: 10.3233/WOR-121585] [PMID]

[5] Wilkins K. Work stress among health care providers. Health Reports. 2007;18(4):82-3. [PMID]

[6] Abbasi M, Janbozorgi M. [Relationship of dimensions of religious orientation with emotional stability (Persian)]. Ravanshenasi Va Din. 2010; 3(3):5-18.

[7] Gardner HE. Intelligence reframed: Multiple Iraellibencies for the $21^{\text {th }}$. New York: Basic Books; 1999.

[8] Khavari K, Abbasi R, Afshari A, Talebi M. [The relationship between spiritual intelligence and emotional responses of nurses in Tehran University's hospitals (Persian)]. Social Welfare Quarterly. 2014; 14(53):165-77.

[9] Rezapour Y, Rezai H, Hosseini S, Mohseni Takalu M. The role of faith in work, religious beliefs, and spirituality in the prediction of job satisfaction among rehabilitation experts. Iranian Rehabilitation Journal. 2017; 14(4):217-22. [DOI:10.18869/nrip. irj.14.4.217]

[10] Bahrami F, Farani A, Younesi J. Role of internal and external religious beliefs in mental health and rate of depression in elderly people. Iranian Rehabilitation Journal. 2007; 5(1):24-9.

[11] Jafari A, Hesampour F. [Predicting life satisfaction based on spiritual intelligence and psychological capital in older people (Persian)]. Iranian Journal of Ageing. 2017; 12(1):90-130. [DOI:10.21859/sija-120190]

[12] Khabaz M, Rezapour-Mirsaleh Y, Abdi K, Safi MH. [A comparative study between the role of religious orientations and personality dimensions in the prediction of coping strategies in mothers of children with and without intellectual disability (Persian)]. Archives of Rehabilitation. 2011;12(2):41-7.

[13] Sa'adati H, Lashani L. [Spiritual intelligence relationship with coping strategies (Persian)]. Archives of Rehabilitation. 2012; 12(Supplementary: Student Articles):75-81.

[14] Ebadi BN, Rahgoi A, Hosseini MA, Fallahi Khoshknab M, Biglarian A. [The correlation between spiritual well-being and hope among nursing students of Medical Sciences Universities and Islamic Azad Universities of Yazd (Persian)]. Journal of Health Promotion Management. 2017; 6(2):8-13.
[15] Rezapour-Mirsaleh Y, Abdi K, Rahgozar M, Reyhani-Kivi S. [Relationship of coping style and self-efficacy with satisfaction of rehabilitation practitioner students with their clinical experiences (Persian)]. Archives of Rehabilitation. 2011; 11(4):47-54.

[16] Raghib M, Siyadat A, Hakiminia B, Ahmedi J. [Validation of the King Spiritual Intelligence Scale (SISRI-24) in Isfahan University students (Persian)]. Journal of Psychology Achievements. 2012; 17(1):141-64.

[17] Ghaffari M, Rezaei A. [Religious commitment and self-efficacy in predicting the amount and type of perceived stress in university students (Persian)]. Journal of Research in Behavioural Sciences. 2000; 9(4):269-78.

[18] Hosseini M, Khoshknab MF, Shahbolaghi FM, Zaheri SM, Soltani P, Khanjani M. The effect of mindfulness program on the perceived stress of family caregivers of elderlies with alzheimer's disease. Journal of Nursing Education. 2016; 4(3):1-7. [DOI:10.21859/ijpn-04031]

[19] Hossein Chari M, Zakeri H. [The effect of studying university majors, religious and art sciences on spiritual intelligence: a trial for validation and measuring reliability of spiritual intelligence scale (Persian)]. Educational Measurement. 2010; 1(1):71-94.

[20] Akbarizadeh F, Bagheri F, Hatami H, Hajivandi A. [Relationship between nurses' spiritual intelligence with hardiness and general health (Persian)]. Journal of Kermanshah University of Medical Sciences (Behbood). 2012; 15(6):466-72.

[21] Noorzad Gharamelki F, Mirnasab M, Ghobari Banab B, Hashemi T. [Relationship between spiritual intelligence and religious coping techniques in Tabriz university students (Persian)]. Paper presented at: The $1^{\text {st }}$ National Conference on Cognitive Science in Education and Training. 23-24 November 2011; Mashhad, Iran.

[22] Crawford J, Henry J. The Depression Anxiety Stress Scales (DASS): normative data and latent structure in a large non-clinical sample. British Journal of Clinical Psychology. 2005; 44(2):22739. [DOI:10.1348/014466505X29657] [PMID]

[23] Soori H, Rahimi M, Mohseni H. [Association between job stress and work-related injuries: A case-control (Persian)]. Iranian Journal of Epidemiology. 2005; 1(3\&4):53-8.

[24] Aghilinejad M, Mohammadi S, Afkari M, Abbaszade Dizaji R. Surveying the association between occupational stress and mental health, personality and life stressful events in Tehran police officers. Research in Medicine. 2007; 31(4):355-60.

[25] Hains A. A stress inoculation training program for adolescents in a high school setting: a multiple baseline approach. Journal of Adolescence. 1992; 15(2):163-75. [DOI:10.1016/01401971[92]90045-7]

[26] Lavasani M, Keyvanzade M, Arjmand N. Spirituality, job stress, organizational commitment, and job satisfaction among nurses in Tehran. Journal of Contemporary Psychology. 2008; 3(2):61-73.

[27] Baezzat F, Sharifzadeh H. Relationship between spiritual intelligence and emotional intelligence with job stress in university employees. Quarterly Journal of Career and Organizational Counseling. 2012; 4(13):55-68. 
[28] Gall T, Charbonneau C, Clarke N, Grant K, Joesph A, Shouldice L. Understanding the nature and role of spirituality in relation to coping and health. Canadian Psychology. 2005; 46(2):88-104. [DOI:10.1037/h0087008]

[29] Beck R. Communion and complaint: Attachment, object-relations, and triangular love perspective on relationship with God. Journal of Psychology \& Theology. 2006; 34(1):43-53. [DOI:10.1 177/009164710603400105]

[30] Laurin K, Kay A, Mosovitch D. On the belief in God: Towards an understanding of the emotional substrates of compensatory control. Journal of Experimental Social Psychology. 2008; 44(6):1559-62. [DOI:10.1016/j.jesp.2008.07.007] 
